\title{
In Vitro Activity of Tedizolid, Dalbavancin, and Ceftobiprole Against Clostridium difficile
}

\author{
Dana Binyamin 1,2, Orna Nitzan²,3, Maya Azrad ${ }^{1}$, Zohar Hamo 1,2, Omry Koren ${ }^{2 \dagger}$ and \\ Avi Peretz ${ }^{1,2 *+}$
}

' Clinical Microbiology Laboratory, Baruch Padeh Medical Center, Poriya, Azrieli Faculty of Medicine, Bar Ilan University, Galilee, Israel, ${ }^{2}$ The Azrieli Faculty of Medicine, Bar Ilan University, Galilee, Israel, ${ }^{3}$ Unit of Infectious Diseases, Baruch Padeh Medical Center, Poriya, Azrieli Faculty of Medicine, Bar Ilan University, Galilee, Israel

OPEN ACCESS

Edited by:

Santi M. Mandal,

Indian Institute of Technology

Kharagpur, India

Reviewed by:

Suman Saha,

Priyamvada Birla Aravind Eye

Hospital, India

Joy Scaria,

South Dakota State University,

United States

*Correspondence:

Avi Peretz

aperetz@poria.health.gov.il

tThese authors have contributed equally to this work.

Specialty section:

This article was submitted to

Antimicrobials, Resistance and Chemotherapy,

a section of the journal

Frontiers in Microbiology

Received: 17 October 2017

Accepted: 24 May 2018

Published: 11 June 2018

Citation:

Binyamin D, Nitzan O, Azrad M, Hamo Z, Koren O and Peretz A (2018) In Vitro Activity of Tedizolid,

Dalbavancin, and Ceftobiprole

Against Clostridium difficile.

Front. Microbiol. 9:1256.

doi: 10.3389/fmicb.2018.01256
Background: Clostridium difficile (C. difficile) is a major nosocomial pathogen that colonizes in the human gut. Recently, the U.S. FDA approved three new antimicrobial agents against gram-positive bacteria: Tedizolid, Dalbavancin, and Ceftobiprole. The efficacy of these antibiotics for treatment of $C$. difficile infection has not been thoroughly examined. The current study aimed to examine the in vitro activity of these antibiotics against $C$. difficile. In addition, to compare between Dalbavancin and Ceftobiprole to antibiotics from the same class: Vancomycin and Ceftriaxone, respectively.

Methods: Eighty-four C. difficile isolates were tested for susceptibility to Tedizolid, Dalbavancin, Ceftobiprole, Vancomycin, and Ceftriaxone by Etest technique in order to determine the minimum inhibitory concentration (MIC).

Results: Upon comparison of the novel antibiotic agents, Dalbavancin demonstrated the lowest $\mathrm{MIC}$ values and ceftobiprole the highest at $\mathrm{MIC}_{50}(0.016,0.38$, and $1.5 \mu \mathrm{g} / \mathrm{mL}$, for Dalbavancin, Tedizolid, and Ceftobiprole, respectively) and $\mathrm{MIC}_{90}(0.03$, 0.78 , and $3.17 \mu \mathrm{g} / \mathrm{mL}$, respectively). Dalbavancin demonstrated significantly lower $\mathrm{MIC}_{50}$ and $\mathrm{MIC}_{90}$ values compared to Vancomycin (0.016 vs. 0.38 and 0.03 vs. 3.5, respectively) $(p<0.001)$ and ceftobiprole had significantly lower MIC values compare to ceftriaxone (1.5 vs. 32 and 3.17 vs. 28.8, respectively) $(p<0.001)$.

Conclusion: Dalbavancin and Tedizolid may play a role as potential therapeutic agents for treatment of $C$. difficile infection. Examination of antibiotic effect on the intestinal microbiome and clinical trials are needed for more accurate results.

Keywords: Clostridium difficile, vancomycin, tedizolid, dalbavancin, ceftobiprole, ceftriaxone

\section{INTRODUCTION}

Clostridium difficile is a gram-positive rod, an obligate anaerobe, spore forming, toxin-producing bacterium that colonizes the human gut (Hall and O'Toole, 1935). The incidence of C. difficile infection (CDI) has increased markedly worldwide since 2000 (Kelly and LaMont, 2008). In Israel, the incidence of $C$. difficile infection in 2016 was 2761 cases per 100,000 patients (Israeli National Center for Infection Control, unpublished data). Disease symptoms include diarrhea and abdominal pain, fever, and increased levels of blood lymphocytes. In severe cases, pseudomembranous colitis, toxic megacolon, or colonic perforation may occur, with high mortality 
rates (Johanesen et al., 2015). The highly resistant spores can survive on surfaces for long periods, and thus be easily transferred from person to person. This occurs mainly in hospitals and longterm care facilities; therefore CDI is a nosocomial infection and has important clinical and financial implications for hospitals (Paredes-Sabja et al., 2014).

The major risk factor for CDI is antibiotic administration, which disrupts the normal intestinal microbiota, leading to spore germination and proliferation of C. difficile. Although nearly all antimicrobial classes have been associated with CDI, clindamycin, third-generation cephalosporins, fluoroquinolones, and penicillins are most commonly associated with this disease (Owens et al., 2008).

Several treatment strategies exist; in some cases, cessation of antibiotics that induced CDI is sufficient for a cure. However, the majority of patients are treated with antibiotics such as metronidazole and Vancomycin (Davies et al., 2011). In severe and recurrent cases, fecal microbiota transplantation, a treatment that restores the normal fecal microbiota, is efficacious (Youngster et al., 2014).

Recently, the U.S. FDA approved three new antimicrobial agents against gram-positive bacteria: Tedizolid, Dalbavancin, and Ceftobiprole. Tedizolid is an oxazolidinone derivate with higher potency than Linezolid, requiring a lower dose for effective result (Im et al., 2011; Prokocimer et al., 2013; Sahm et al., 2015). Tedizolid is highly active against gram-positive bacteria, and can be used for the treatment of acute bacterial skin and skin structure infections (Ferrández et al., 2017). This antibiotic binds to the 50S subunit of the bacterial ribosome and inhibits protein synthesis (Shaw et al., 2008).

Dalbavancin is a bactericidal lipoglycopeptide antibiotic that inhibits cell wall synthesis. Similar to Vancomycin, it belongs to the glycopeptide antibiotic group and is an efficient treatment for infections caused by Vancomycin-resistant strains (Tatarkiewicz et al., 2016). In vitro Dalbavancin activity against various grampositive species, including $C$. difficile, was more potent compared to Vancomycin (Goldstein et al., 2003; Huband et al., 2016; Tatarkiewicz et al., 2016).

Ceftobiprole is a new generation of cephalosporin with broadspectrum activity against gram-positive and gram-negative bacteria (Ednie et al., 2007). Ceftobiprole is a $\beta$-lactam antibacterial with bactericidal activity (Murthy and SchmittHoffmann, 2008).

The current study aimed to examine the in vitro activity of Tedizolid, Dalbavancin, and Ceftobiprole against C. difficile by detecting the minimum inhibitory concentration (MIC). In addition, we also compared Dalbavancin and Ceftobiprole to Vancomycin and Ceftriaxone, respectively, antibiotics from the same class.

\section{MATERIALS AND METHODS}

\section{Study Population}

Patients diagnosed with $C$. difficile infection at the Baruch Padeh Medical Center, Poriya in northern Israel, were enrolled in the study from January 2015 to May 2017. The identification of CDI was performed by stool examination for toxigenic $C$. difficile at the Clinical Microbiology Laboratory by Xpert ${ }^{\circledR}$ C. difficile Assay (Cepheid, Solna, Sweden), performed on Cepheid GeneXpert ${ }^{\circledR}$ Systems. This is a qualitative in vitro real-time PCR for the rapid identification of $C$. difficile. The study was approved by the Poriya Baruch Padeh Medical Center Helsinki Committee without the need for patients to sign an informed consent form because the study deals with microbial isolates and the results of the study do not affect the patients.

\section{Bacteria Isolation and Identification}

For this purpose, $0.5 \mathrm{~mL}$ of liquid feces was suspended in $4.5 \mathrm{~mL}$ physiological solution. Fifty $\mu \mathrm{L}$ of the suspension were inoculated on a selective CHRomagar medium; chromID ${ }^{\mathrm{TM}}$ C. difficile (bioMérieux, France) and then incubated at $37^{\circ} \mathrm{C}$ in anaerobic conditions (GasPakTM EZ, BD, United States) for 48 h. C. difficile colonies appear as asymmetric and black-colored colonies. Final identification was done by matrix-assisted laser desorption ionization-time of flight mass spectrometry (MALDITOF MS)-based technology using the Bruker Biotyper system (Bruker, United States).

\section{Antibiotic Susceptibility Tests}

Antibiotic susceptibility tests were performed by the Etest technique in order to determine the minimum inhibitory concentration (MIC), which is the lowest antibiotic concentration that inhibits bacterial growth. To this end, bacteria colonies were suspended in saline, generating turbidity of 0.5 McFarland. The suspensions were seeded on Mueller Hinton $+5 \%$ Sheep Blood agar plate (HyLaboratories, Rehovot, Israel). Then, a gradient Etest strip (Liofilchem, Italy) for each antibiotic was added to each plate and incubated at $37^{\circ} \mathrm{C}$ in anaerobic conditions for $48 \mathrm{~h}$. After incubation, the susceptibility breakpoint was determined as the lowest concentration at which no bacterial growth was detected. Additionally, $\mathrm{MIC}_{90}$ and $\mathrm{MIC}_{50}$ were calculated as the MICs at which $90 \% / 50 \%$ of the isolates tested are inhibited. Etest procedures were done for Tedizolid, Dalbavancin, and Ceftobiprole, as well as for Vancomycin and Ceftriaxone for comparison to Dalbavancin and Ceftobiprole, respectively.

\section{Statistical Analysis}

Differences between the $\mathrm{MIC}_{50}$ or $\mathrm{MIC}_{90}$ of Tedizolid, Dalbavancin, and Ceftobiprole were analyzed by one-way analysis of variance (ANOVA), with Benferroni's post hoc test.

Differences between the $\mathrm{MIC}_{50}$ or $\mathrm{MIC}_{90}$ of Ceftobiprole and ceftriaxone as well as the differences between the $\mathrm{MIC}_{50}$ or $\mathrm{MIC}_{90}$ of Dalbavancin and Vancomycin, were analyzed by Wilcoxon matched-pairs signed rank test.

Statistical significance was defined by $p<0.05$.

\section{RESULTS}

Eighty-four C. difficile isolates were tested for susceptibility to Tedizolid, Dalbavancin, and Ceftobiprole. $\mathrm{MIC}_{50}$ and $\mathrm{MIC}_{90}$ 
TABLE 1 | Susceptibility test (MICs) of C. difficile isolates.

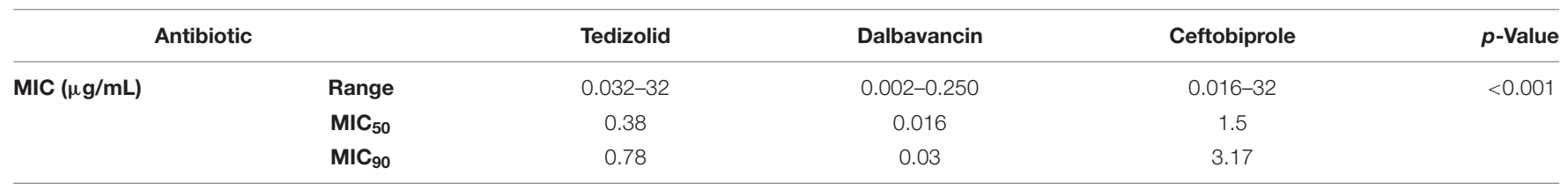

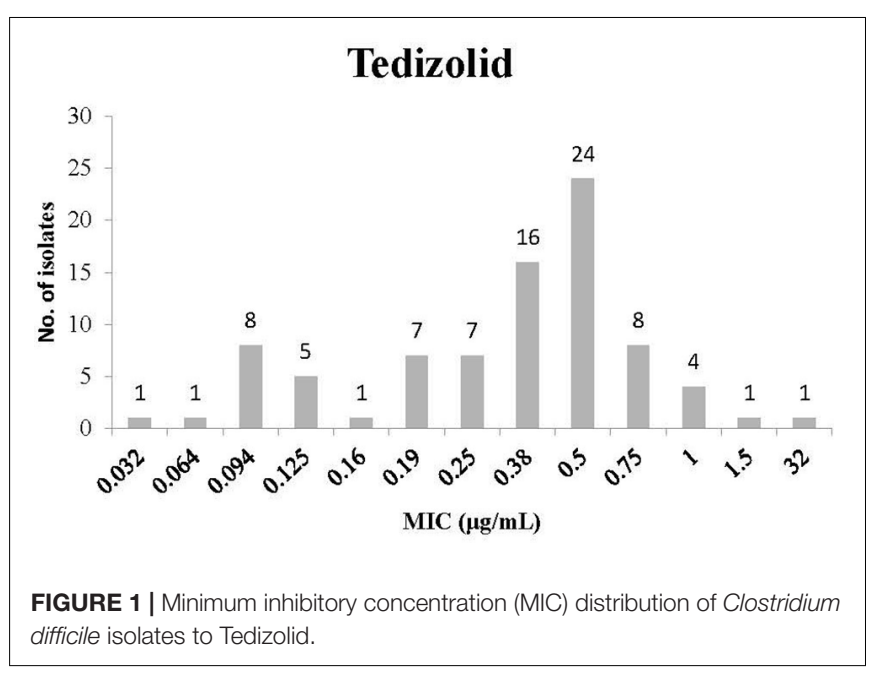

results are presented in Table 1 . The $\mathrm{MIC}_{50}$ and $\mathrm{MIC}_{90}$ results of each antibiotic were different from the other antibiotic agents $(p<0.001)$. Dalbavancin demonstrated low MIC results compared to Tedizolid and Ceftobiprole, for $\mathrm{MIC}_{50}(0.016$, 0.38 , and $1.5 \mu \mathrm{g} / \mathrm{mL}$, respectively) and $\mathrm{MIC}_{90}(0.03,0.78$, and $3.17 \mu \mathrm{g} / \mathrm{mL}$, respectively). Ceftobiprole was the antibiotic with the highest MIC values compared to the two other antibiotics.

The distribution of MIC for Tedizolid is presented in Figure 1. Most isolates had an MIC of $0.5 \mu \mathrm{g} / \mathrm{mL}$ (24 isolates) or $0.38 \mu \mathrm{g} / \mathrm{mL}$ (16 isolates). In addition, some of the isolates had low MIC values (for instance 8 isolates with $0.094 \mu \mathrm{g} / \mathrm{mL}$ and 5 isolates with $0.125 \mu \mathrm{g} / \mathrm{mL}$ ). However, other isolates had higher MIC values (for instance 1 isolate with an MIC of $32 \mu \mathrm{g} / \mathrm{mL}$ and 8 isolates with an MIC of $0.75 \mu \mathrm{g} / \mathrm{mL}$ ).

Figure 2 presents the MIC distribution for Dalbavancin. Overall, Dalbavancin susceptibility results demonstrated low MIC values. Most isolates had an MIC of $0.016 \mu \mathrm{g} / \mathrm{mL}(13$ isolates) and 0.012 or $0.023 \mu \mathrm{g} / \mathrm{mL}$ (12 isolates).

Minimum inhibitory concentration distribution for Ceftobiprole is presented in Figure 3. Most isolates had an MIC of $1.5 \mu \mathrm{g} / \mathrm{mL}$ (21 isolates) or $2 \mu \mathrm{g} / \mathrm{mL}$ (15 isolates). Many isolates had high MIC values; for instance 8 isolates with MIC of $3 \mu \mathrm{g} / \mathrm{mL}, 6$ with $4 \mu \mathrm{g} / \mathrm{mL}$, and 3 with $32 \mu \mathrm{g} / \mathrm{mL}$.
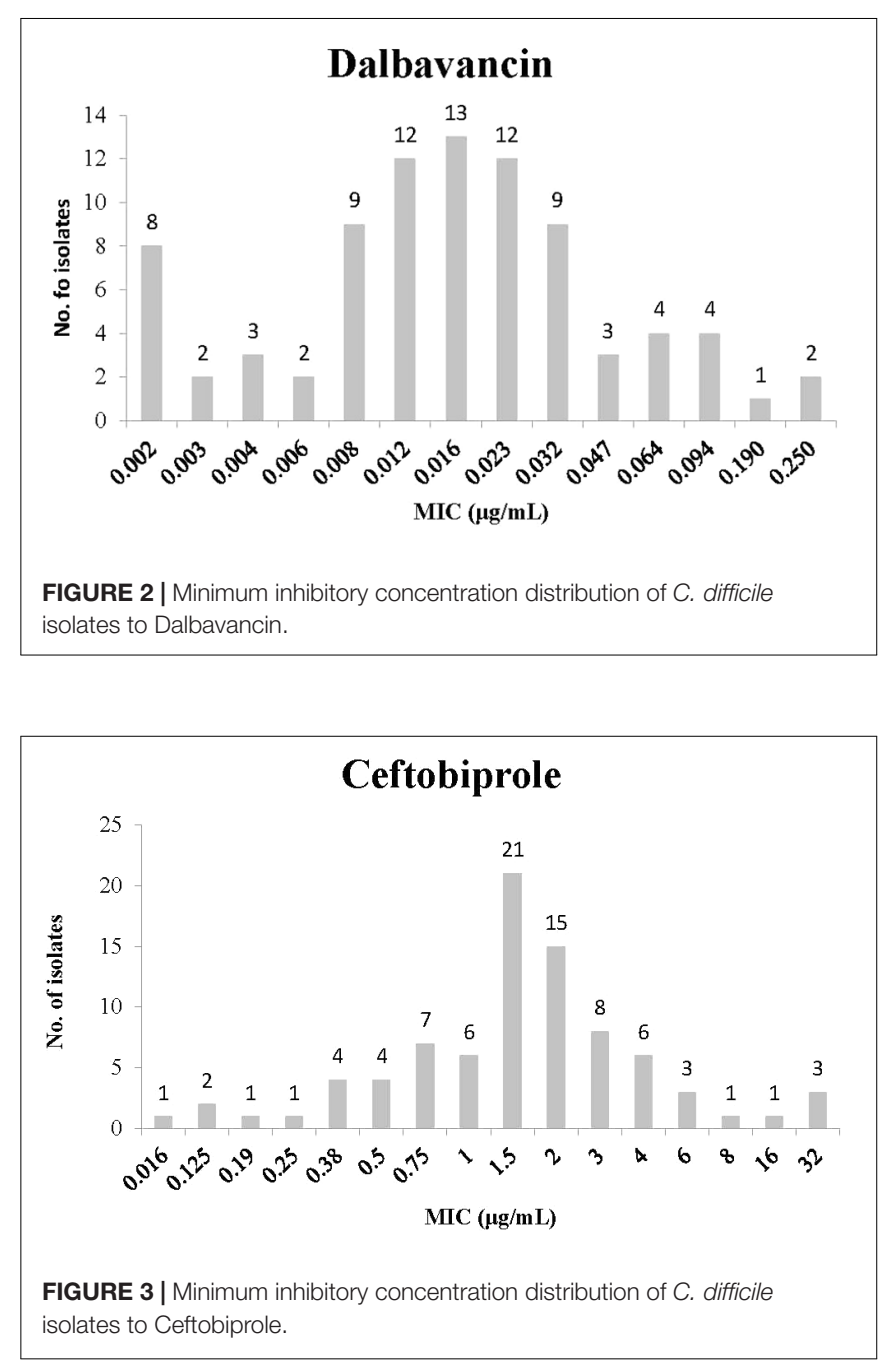

We compared the susceptibility of antibiotics from the same antibiotic group (Table 2). In a comparison of the $\mathrm{MIC}_{50}$ and $\mathrm{MIC}_{90}$ between Dalbavancin and Vancomycin, Dalbavancin showed significantly lower MIC values ( 0.016 vs. 0.38 and 0.03 vs. 3.5 , respectively, $p<0.001$ ).

TABLE 2 | Comparison between susceptibility tests (MICs) of antibiotics in the same antibiotic group.

\begin{tabular}{cccccccc}
\hline Antibiotic & & Dalbavancin & Vancomycin & $\boldsymbol{p}$-Value & Ceftobiprole & Ceftriaxone & $\boldsymbol{p}$-Value \\
\hline MIC $(\mu \mathbf{g} / \mathbf{m L})$ & Range & $0.002-0.250$ & $0.016-256$ & $<0.001$ & $0.016-32$ & $0.38-32$ \\
& MIC $_{\mathbf{5 0}}$ & 0.016 & 0.38 & & 1.5 & 32 \\
& MIC $_{\mathbf{9 0}}$ & 0.03 & 3.5 & & 3.17 & 28.8
\end{tabular}


In comparison of the $\mathrm{MIC}_{50}$ and $\mathrm{MIC}_{90}$ between Ceftobiprole and Ceftriaxone, Ceftobiprole had significantly lower MIC values (1.5 vs. 32 and 3.17 vs. 28.8 , respectively, $p<0.001$ ).

\section{DISCUSSION}

Antibiotic administration is the main risk factor for CDI, even though treatment of the disease includes antibiotic therapy. Despite the efficacy of the conventional antibiotics for treating the disease, treatment failure, recurrence, and antibiotic resistance are reported (Adler et al., 2015; Freeman et al., 2015). Therefore, it is important to identify new antibiotics that may potentially be used to treat CDI.

The current study examined the MIC of three recently approved antibiotics: Tedizolid, Dalbavancin, and Ceftobiprole. These antibiotics are affective against gram-positive bacteria such as methicillin resistant Staphylococcus aureus and Vancomycin resistant Enterococci (Ednie et al., 2007; Im et al., 2011; Huband et al., 2016). However, antibiotic efficacy against $C$. difficile has not been thoroughly examined.

Of all three antibiotics, Dalbavancin was found to be the most effective antibiotic with high prevalence of isolates with low MIC values. In comparison to Vancomycin, another lipoglycopeptide antibiotic, Dalbavancin, had significantly lower MIC values for C. difficile. These results are different from those found in a previous study, where higher MIC levels for Dalbavancin and lower MIC levels for Vancomycin were demonstrated, compared with our findings, but similar to what we found - MIC values for Dalbavancin were lower than those of Vancomycin (Goldstein et al., 2003). Vancomycin is considered first line treatment of moderate to severe CDI (Davies et al., 2011). Different studies reported resistance rates of 7.4-47\% to Vancomycin, treatment failure in $14.2 \%$ of cases, and recurrence rate of $24.0 \%$ (Adler et al., 2015; Freeman et al., 2015; Tkhawkho et al., 2017). Due to the presence of Vancomycin-resistant strains and recurrence cases, Dalbavancin might be used as treatment for CDI. Further research is required, mainly clinical trials and examination of antibiotic effect on the intestinal microbiome.

Ceftobiprole had the highest MIC values for $C$. difficile compared to the other examined antibiotics, although these MIC values were lower than those found in another study (Ednie et al., 2007). In comparison to another broad spectrum cephalosporin, ceftriaxone, C. difficile isolates had significantly

\section{REFERENCES}

Adler, A., Miller-Roll, T., Bradenstein, R., Block, C., Mendelson, B., Parizade, M., et al. (2015). A national survey of the molecular epidemiology of Clostridium difficile in Israel: the dissemination of the ribotype 027 strain with reduced susceptibility to vancomycin and metronidazole. Diagn. Microbiol. Infect. Dis. 83, 21-24. doi: 10.1016/j.diagmicrobio.2015.05.015

Bäckström, T., Panagiotidis, G., Beck, O., Asker-Hagelberg, C., Rashid, M.-U., Weintraub, A., et al. (2010). Effect of ceftobiprole on the normal human intestinal microflora. Int. J. Antimicrob. Agents 36, 537-541. doi: 10.1016/j. ijantimicag.2010.07.021

Bensaci, M., and Sahm, D. (2017). Surveillance of tedizolid activity and resistance: in vitro susceptibility of Gram-positive pathogens collected over 5 years from lower MIC values. Cephalosporins have poor in vitro activity against C. difficile (Wilcox et al., 2016). Some cephalosporins even promote $C$. difficile spore germination, proliferation, and toxin production (Wilcox et al., 2016). However, a study in a mouse model that tested Ceftobiprole's effect on $C$. difficile found that Ceftobiprole does not promote the growth of C. difficile or toxin production, in contrast to Ceftazidime, Cefotaxime, and Ceftriaxone (Nerandzic and Donskey, 2011). Another study that investigated the effect of Ceftobiprole administration on normal intestinal microbiota found that it had no significant ecological impact on the human intestinal microbiota (Bäckström et al., 2010). Consequently, Ceftobiprole can be used for CDI treatment and when administered for other indications may be associated with a reduced risk for CDI compared with other cephalosporins.

We also found a high prevalence of isolates with low MIC values to Tedizolid. This antibiotic was shown to have high activity against gram-positive bacteria, mainly skin pathogens, with low MIC rates (Bensaci and Sahm, 2017; Ferrández et al., 2017). Ours is the first study that examines the effect of Tedizolid on $C$. difficile. In a clinical trial, fewer patients who had received Tedizolid suffered from gastrointestinal side effects (16\%) than those who received Linezolid (23\%) (Shorr et al., 2015). Further research is warranted to determine whether Tedizolid is effective against $C$. difficile.

\section{CONCLUSION}

The activity of three novel antibiotics - Tedizolid, Dalbavancin, and Ceftobiprole - against $C$. difficile was examined. Dalbavancin and Tedizolid may be potential therapeutic agents for the treatment of CDI. Clinical trials are needed to confirm the laboratory experiments, as well as studies that examine the effect of these antibiotics on the intestinal microbiome to ensure they are not a risk factor for CDI.

\section{AUTHOR CONTRIBUTIONS}

$\mathrm{DB}, \mathrm{ON}, \mathrm{OK}$, and AP designed the study and interpreted the data. $\mathrm{AP}, \mathrm{MA}$, and $\mathrm{ON}$ drafted the manuscript. DB, $\mathrm{ZH}, \mathrm{MA}$, and AP performed laboratory work. All authors read and approved the final manuscript.

the United States and Europe. Diagn. Microbiol. Infect. Dis. 87, 133-138. doi: 10.1016/j.diagmicrobio.2016.10.009

Davies, A. H., Roberts, A. K., Shne, C. C., and Acharya, K. R. (2011). Super toxins from a super bug: structure and function of Clostridium difficile toxins. Biochem. J. 436, 517-526. doi: 10.1042/BJ20110106

Ednie, L., Shapiro, S., and Appelbaum, P. C. (2007). Antianaerobe activity of ceftobiprole, a new broad-spectrum cephalosporin. Diagn. Microbiol. Infect. Dis. 58, 133-136. doi: 10.1016/j.diagmicrobio.2006.10.015

Ferrández, O., Urbina, O., and Grau, S. (2017). Critical role of tedizolid in the treatment of acute bacterial skin and skin structure infections. Drug Des. Devel. Ther. 11, 65-82. doi: 10.2147/DDDT.S84667

Freeman, J., Vernon, J., Morris, K., Nicholson, S., Todhunter, S., Longshaw, C., et al. (2015). Pan-European longitudinal surveillance of antibiotic resistance 
among prevalent Clostridium difficile ribotypes. Clin. Microbiol. Infect. 21, 248.e9-248.e16. doi: 10.1016/j.cmi.2014.09.017

Goldstein, E. J., Citron, D. M., Merriam, C. V., Warren, Y., Tyrrell, K., and Fernandez, H. T. (2003). In vitro activities of dalbavancin and nine comparator agents against anaerobic gram-positive species and Corynebacteria. Antimicrob. Agents Chemother. 47, 1968-1971. doi: 10.1128/AAC.47.6.1968-1971.2003

Hall, I. C., and O'Toole, E. (1935). Intestinal flora in new-born infants with a description of a new pathogenic anaerobe, Bacillus difficilis. Am. J. Dis. Child. 49, 390-402. doi: 10.1001/archpedi.1935.01970020105010

Huband, M. D., Castanheira, M., Farrell, D. J., Flamm, R. K., Jones, R. N., Sader, H. S., et al. (2016). In vitro activity of dalbavancin against multidrug-resistant Staphylococcus aureus and streptococci from patients with documented infections in Europe and surrounding regions (2011-2013). Int. J. Antimicrob. Agents 7, 495-499. doi: 10.1016/j.ijantimicag.2016.04.009

Im, W. B., Choi, S. H., Park, J. Y., Choi, S. H., Finn, J., and Yoon, S. H. (2011). Discovery of torezolid as a novel 5-hydroxymethyl-oxazolidinoneantibacterial agent. Eur. J. Med. Chem. 46, 1027-1039. doi: 10.1016/j.ejmech.2011. 01.014

Johanesen, P. A., Mackin, K. E., Hutton, M. L., Awad, M. M., Larcombe, S., and Amy, J. M., et al. (2015). Disruption of the gut microbiome: Clostridium difficile infection and the threat of antibiotic resistance. Genes 6, 1347-1360. doi: 10.3390/genes6041347

Kelly, C. P., and LaMont, J. T. (2008). Clostridium difficile-more difficult than ever. N. Engl. J. Med. 359, 1932-1940. doi: 10.1056/NEJMra0707500

Murthy, B., and Schmitt-Hoffmann, A. (2008). Pharmacokinetics and pharmacodynamics of ceftobiprole, an anti-MRSA cephalosporin with broad-spectrum activity. Clin. Pharmacokinet. 47, 21-33. doi: 10.2165/00003088-200847010-00003

Nerandzic, M. M., and Donskey, C. J. (2011). Effect of ceftobiprole treatment on growth of and toxin production by Clostridium difficile in cecal contents of mice. Antimicrob. Agents Chemother. 55, 2174-2177. doi: 10.1128/AAC.01 $612-10$

Owens, R. C., Donskey, C. J., Gaynes, R. P., Loo, V. G., and Muto, C. A. (2008). Antimicrobial-associated risk factors for Clostridium difficile infection. Clin. Infect. Dis. 46(Suppl. 1), S19-S31. doi: 10.1086/521859

Paredes-Sabja, D., Shen, A., and Sorg, J. A. (2014). Clostridium difficile spore biology: sporulation, germination, and spore structural proteins. Trends Microbiol. 22, 406-416. doi: 10.1016/j.tim.2014.04.003

Prokocimer, P., De Anda, C., Fang, E., Mehra, P., and Das, A. (2013). Tedizolid phosphate vs linezolid for treatment of acute bacterial skin and skin structure infections: the ESTABLISH-1 randomized trial. JAMA 309, 559-569. doi: 10.1001/jama.2013.241

Sahm, D. F., Deane, J., Bien, P. A., Locke, J. B., Zuill, D. E., Shaw, K. J., et al. (2015). Results of the surveillance of tedizolid activity and resistance program: in vitro susceptibility of Gram-positive pathogens collected in 2011 and 2012 from the United States and Europe. Diagn. Microbiol. Infect. Dis. 81, 112-118. doi: 10.1016/j.diagmicrobio.2014.08.011

Shaw, K. J., Poppe, S., Schaadt, R., Brown-Driver, V., Finn, J., Pillar, C. M., et al. (2008). In vitro activity of TR-700, the antibacterial moiety of the prodrug TR-701, against linezolid-resistant strains. Antimicrob. Agents Chemother. 52, 4442-4447. doi: 10.1128/AAC.00859-08

Shorr, A. F., Lodise, T. P., Corey, G. R., De Anda, C., Fang, E., Das, A. F., et al. (2015). Analysis of the phase 3 ESTABLISH trials of tedizolid versus linezolid in acute bacterial skin and skin structure infections. Antimicrob. Agents Chemother. 59, 864-871. doi: 10.1128/AAC.03688-14

Tatarkiewicz, J., Staniszewska, A., and Bujalska-Zadrożny, M. (2016). New agents approved for treatment of acute staphylococcal skin infections. Arch. Med. Sci. 12, 1327-1336. doi: 10.5114/aoms.2016.59838

Tkhawkho, L., Nitzan, O., Pastukh, N., Brodsky, D., Jackson, K., and Peretz, A. (2017). Antimicrobial susceptibility of Clostridium difficile isolates in Israel. J. Glob. Antimicrob. Resist. 10, 161-164. doi: 10.1016/j.jgar.2017.04.005

Wilcox, M. H., Chalmers, J. D., Nord, C. E., Freeman, J., and Bouza, E. (2016). Role of cephalosporins in the era of Clostridium difficile infection. J. Antimicrob. Chemother. 2, 1-18.

Youngster, I., Sauk, J., Pindar, C., Wilson, R. G., Kaplan, J. L., Smith, M. B., et al. (2014). Fecal microbiota transplant for relapsing Clostridium difficile infection using a frozen inoculum from unrelated donors: a randomized, open-label, controlled pilot study. Clin. Infect. Dis. 58, 1515-1522. doi: 10.1093/cid/ciu135

Conflict of Interest Statement: The authors declare that the research was conducted in the absence of any commercial or financial relationships that could be construed as a potential conflict of interest.

Copyright (๑) 2018 Binyamin, Nitzan, Azrad, Hamo, Koren and Peretz. This is an open-access article distributed under the terms of the Creative Commons Attribution License (CC BY). The use, distribution or reproduction in other forums is permitted, provided the original author(s) and the copyright owner are credited and that the original publication in this journal is cited, in accordance with accepted academic practice. No use, distribution or reproduction is permitted which does not comply with these terms. 